\title{
Prácticas sexuales y uso del preservativo en adolescentes escolarizados de Medellín, 2014
}

Karen Stefanía Restrepo Velásquez ${ }^{1}$, Anamaria Toro Taborda', Jaiberth Antonio Cardona-Arias ${ }^{2}$

\section{Resumen}

Introducción: La adolescencia es un periodo de grandes cambios físicos, psicológicos, emocionales y sociales que incrementan el riesgo sexual.

Objetivo: Estimar la prevalencia de prácticas sexuales de riesgo y su asociación con el uso del preservativo en adolescentes escolarizados en instituciones públicas de Medellín.

Material y métodos: Estudio de prevalencia en 3.453 adolescentes seleccionados aleatoriamente. Se empleó fuente primaria de información. Los análisis se basaron en proporciones, intervalos de confianza, Prueba Z, Chi cuadrado, t Student, razones de prevalencia y regresión logística.

Resultados: $39,7 \%$ fueron hombres, $50,7 \%$ de estrato socioeconómico bajo; la principal fuente de información en sexualidad fue internet, $42,6 \%$ viven en familias nucleares. El $54,6 \%$ ha iniciado su vida sexual, con edad media de inicio de 14 años. La proporción de adolescentes que han tenido relaciones sexuales fue estadísticamente mayor en hombres, de adolescencia tardía, provenientes de familias monoparentales y en quienes buscan información sobre salud sexual en internet o amigos. La frecuencia de uso del condón fue 66,3\%; $58,7 \%$ lo utilizan de forma incorrecta y sólo $38,3 \%$ lo usa en todas sus relaciones sexuales.

Conclusión: El elevado riesgo sexual, la elevada frecuencia de mitos sobre el condón y la identificación de grupos de mayor vulnerabilidad sexual, constituyen resultados de gran utilidad para formular, implementar o evaluar las políticas públicas relacionadas con la adolescencia y la salud sexual y reproductiva.

1 Grupo de Investigación Salud y Sostenibilidad. Escuela de Microbiología, Universidad de Antioquia.

2 Microbiólogo y Bioanalista, MSc Epidemiología. Docente Universidad de Antioquia UdeA, Calle 70 No. 52-21, Medellín, Colombia. Docente Facultad de Medicina Universidad Cooperativa de Colombia.

Correspondencia:

Jaiberth Antonio Cardona Arias

झ] jaiberthcardona@gmail.com 
Palabras clave: Prácticas; Sexual, Adolescentes; Métodos de planificación; Colombia.

\section{Sexual practices and condom use among adolescent students of Medellín, 2014}

\section{Abstract}

Introduction: The adolescence is a time of great physical, psychological, emotional and social changes that increase sexual risk.

Objective: To estimate the prevalence of unsafe sex and its association with condom use among adolescents students in public institutions of Medellin.

Methods: Cross sectional study in 3,453 adolescents randomly selected. Primary source of information was used. Analyses were based on proportions, confidence intervals, Z test, Chi square, Student $\mathrm{t}$, prevalence ratios and logistic regression.

Results: $39.7 \%$ were men, $50.7 \%$ of low socioeconomic status; the main source of information on sexuality was internet, $42.6 \%$ live in nuclear families. $54.6 \%$ had initiated sexual life, mean age of onset was 14 years. The proportion of adolescents who have had sex was statistically higher in men, late adolescence, from single-parent families and those seeking sexual health information on the internet or friends. The frequency of condom use was $66.3 \% ; 58.7 \%$ use it incorrectly and only $38.3 \%$ use it in all sexual relationships.

Conclusion: The high sexual risk, high frequency of myths about condoms and identifying groups at higher sexual vulnerability, are results useful for the formulating, implementing and evaluating of public policies related with the adolescence and the sexual and reproductive health.

Keywords: Practices; Sexual; Adolescents; Planning techniques; Colombia. 


\section{Introducción}

La adolescencia es una etapa de la vida comprendida entre los 13 y los 19 años, en la que hombres y mujeres experimentan cambios físicos, psicológicos, emocionales y sociales ${ }^{1}$. Esta etapa trae consigo algunos factores protectores y otros que ponen en peligro la vida y la salud de las personas. Entre los factores protectores se pueden enunciar el tener hogares nucleares completos, una buena comunicación interpersonal, alta autoestima, una relación emocional estable con al menos uno de sus padres, un ambiente educativo abierto, práctica de deporte, pertenecer a grupos que aporten al desarrollo personal y tener un proyecto de vida². Entre los factores de riesgo se encuentran la pobreza, el tipo de escolaridad, la discriminación étnica, el acceso a tabaco, alcohol y otras drogas; los conocimientos inadecuados o deficientes frente a la salud sexual y reproductiva, y el inicio de la vida sexual sin uso de métodos de barrera (condón) 3 .

Durante la adolescencia inicia la búsqueda de identidad y orientación sexual, y es una etapa caracterizada por el comienzo de la vida sexual, esto hace que se incrementen las condiciones de vulnerabilidad de este grupo frente a las Infecciones de Transmisión Sexual (ITS), puesto que aunado a la experimentación sexual, se incrementa el número de parejas sexuales y de coitos sin protección ${ }^{4}$. A esto se suma el hecho que los adolescentes presentan una baja percepción de su riesgo frente a diferentes situaciones sanitarias, al considerar que los problemas de salud afectan a los demás; probablemente como consecuencia de una baja capacitación sobre la promoción de su salud y la prevención de enfermedades, o por prejuicios, tabúes, barreras culturales u otros obstáculos que presentan los adolescente al intentar cuidar de sí mismos ${ }^{5}$.

En lo referido a salud sexual y reproductiva, en algunas familias se cohíben de hablar sobre estos temas y las dudas son resueltas por fuentes externas a la familia que quizás no le brindan al adolescente la mejor información, lo que puede derivar en actitudes y prácticas erróneas frente a su vida sexual. Por su parte, la educación sexual en los colegios no ha tenido el impacto esperado, lo que se refleja en que el adolescente muchas veces practica su sexualidad bajo esquemas de riesgo que derivan en el aumento de embarazos no deseados en niñas y adolescentes y el incremento de ITS en este grupo; lo que implica que los adolescentes escolarizados, a pesar de las iniciativas institucionales por fomentar una buena salud sexual, siguen expuestos a múltiples riesgos sexuales ${ }^{5}$.

En este contexto, el condón ha sido referido como el método de planificación más utilizado por adolescentes; sin embargo, la mayoría de veces ha estado ligado al control de natalidad, olvidando que es el método de barrera más importante para evitar las ITS, esto se corrobora aún más al aludir que las mujeres que utilizan otros métodos de planificación se sienten protegidas y no son conscientes del riesgo que tienen frente a ITS, afianzando una vez, que lo central es evitar el embarazo y no protegerse contra las ITS 4 . Además, algunos autores han indicado que las relaciones anales y buco genitales se han convertido en una práctica frecuente en los jóvenes, probablemente por temor al embarazo, desconociendo el riesgo de contraer ITS por estas vías y la necesidad de usar el preservativo como método preventivo ${ }^{6}$.

Es importante resaltar que la mayoría de los jóvenes tienen conocimientos sobre métodos de planificación, información que ha sido suministrada por las instituciones educativas y sus familias; sin embargo, la adhesión a éstos ha sido baja; como evidencian diferentes estudios que han documentado una baja frecuencia del uso correcto del condón (atribuible al desconocimiento de los riesgos que con lleva) y cifras elevadas de ITS y embarazos ${ }^{5,7}$. 
En el ámbito internacional, algunos reportes previos han indicado que el $47 \%$ de los estudiantes alguna vez en su vida han tenido relaciones sexuales y el $33 \%$ de ellos manifiestan ser sexualmente activos; lo preocupante de estas cifras es que sólo el 60,2\% indicaron haber utilizado el condón en su última relación sexual ${ }^{8}$. En el ámbito nacional, los estudios demuestran que el $80 \%$ de los adolescentes escolares en su primera relación sexual no utilizaron el condón a pesar de haber recibido información con anterioridad sobre este método ${ }^{9}$.

Sumado a lo expuesto, diversos estudios han manifestado que el conocimiento en sexualidad sigue siendo muy bajo a pesar de los planes y programas que se han implementado para resolver esta problemática, como la creación de normativas que a obliga a colegios públicos y privados a construir planes de educación sexual desde 19929,10. En Colombia han sido pocos los estudios que han evaluado el riesgo sexual en los adolescentes escolarizados y su relación con el uso del preservativo, entre estos destaca el realizado por González donde se estimó la relación entre los conocimientos, actitudes y prácticas de riesgo sobre la sexualidad en adolescentes de 12 colegios públicos de la localidad de Suba ${ }^{9}$. Con relación al uso y creencias del preservativo, el grupo de Romero realizó una investigación en el centro de Medellín en el año 2005, observando niveles bajos de uso"11; Alvis Nelson además de mostrar un uso limitado informó una alta prevalencia de ITS ${ }^{12}$. Aunado a esto, el estudio de Gómez reportó que sólo el 19,3\% de 516 estudiantes de secundaria en Pereira usó condón en su primera relación sexual y el $25,6 \%$ no utiliza condón en ninguna de sus relaciones sexuales ${ }^{13}$.

Aunado al hecho de ser muy pocas las investigaciones que en Colombia y concretamente en Medellín se han desarrollado sobre esta temática, se debe tener presente que la mayoría se han desarrollado en muestras por conveniencia que no representan adecuadamente la situación de la ciudad ni permiten identificar los grupos de mayor vulnerabilidad en este tema. Por lo anterior, se realizó un estudio con el objetivo de estimar la prevalencia de prácticas sexuales de riesgo y su asociación con el uso del preservativo, en los adolescentes escolarizados en instituciones educativas de Medellín, 2014.

\section{Material y métodos}

\section{Tipo de estudio}

Descriptivo transversal.

\section{Sujetos de estudio}

Adolescentes que cumplieran los criterios de inclusión de estar escolarizado en Instituciones Educativas Públicas de Medellín, entre los grados 10 y 11, habitantes del área urbana y rural, de ambos sexos, de varios estratos sociales. Los criterios de exclusión fueron personas con alteraciones mentales que pudiesen generar pérdida de información por sesgo de memoria, quienes durante la recolección de la información rechazaron su participación en el estudio, sujetos que al momento de la encuesta se encontraban bajo el influjo de alucinógenos y quienes exigían remuneración para participar del estudio; estos criterio fueron aplicados por el coordinador de curso o psicólogo de la institución educativa.

\section{Cálculo del tamaño de la muestra y muestreo}

Se tomó una población de referencia de 200.000 estudiantes, confianza del 95\%, proporción de prácticas sexuales de $50 \%$, error de muestreo $2 \%$ y corrección por pérdidas del 20\%; con ello la muestra fue de 2848. La muestra final se incrementó a 3.453 individuos debido a dos razones: i) las instituciones de la ciudad tienen entre 150 y 350 estudiantes en décimo y undécimo, pero en tres colegios seleccionados aleatoriamente se encontraron más de 500 , ii) en el muestreo probabilístico sólo se debía 
incluir un corregimiento pero debido a la diversidad socioeconómica y demográficas que existen entre los cinco existentes, en la muestra se incluyeron dos instituciones más para representar tal diversidad en este estrato.

El muestreo fue probabilístico bietápico, en la primera etapa se realizó un muestreo estratificado con asignación proporcional, donde los estratos estuvieron conformados por las siete zonas en que se divide la ciudad y en cada una se hizo una selección aleatoria proporcional de las instituciones educativas. En la segunda etapa, al interior de cada zona se seleccionó de forma aleatoria la(s) Institución(es) Educativa(s) participantes según listado de la Secretaría de Educación y de ésta(s) se encuestó la totalidad de estudiantes que cumplían los criterios de inclusión y que no aplicaban para la exclusión.

\section{Recolección de la información:}

Se empleó fuente de información primaria, la cual consistió en una encuesta estructurada con preguntas dicótomas, politómicas y algunas abiertas sobre comportamientos sexuales y uso del condón, a lo que se sumó información demográfica y socioeconómica de cada estudiante. El tipo de familia se categorizó en nuclear cuando el adolescente vive con ambos padres y hermanos, monoparentales para los adolescentes que sólo viven con el padre o la madre, extensa para quienes conviven con primos abuelos u otro tipo de familiares, y en la categoría de "otro" se ubicaron los adolescente que no viven con familiares.

Para el control de sesgos se realizó prueba piloto en un $10 \%$ de la muestra con el fin de evaluar la versión definitiva y el desempeño de los encuestadores; la encuesta fue aplicada por encuestadores capacitados en asuntos relacionados con el contenido del instrumento, técnicas de acercamiento al encuestado, aspectos éticos, condiciones que debía reunir el lugar donde se realizó la encuesta y revisión de esta antes de entregarla para la digitación, su aplicación fue individual, asistida y anónima. A la encuesta se le realizó validación de apariencia por parte de dos epidemiólogos, dos microbiólogos, un psicólogo y 20 estudiantes de décimo y undécimo, con el fin de evaluar su aplicabilidad y aceptabilidad. Además, para evitar el sesgo de información inherente a los encuestados se aplicaron de forma rigurosa los criterios de exclusión.

\section{Análisis de la información:}

Las condiciones demográficas, económicas y sociales de los sujetos del estudio y la prevalencia de las prácticas sexuales de riesgo se describieron con proporciones con sus intervalos de confianza y medidas de resumen. La comparación de la prevalencia de estudiantes que han iniciado su vida sexual con las variable dicotómicas se hizo a través de la prueba Z de comparación de proporciones dado el cumplimiento del supuesto de normalidad (aproximación de la distribución binomial a la normal $n p \geq 5$ ), con las politómicas a través de la prueba chi cuadrado de Pearson y con las cuantitativas a través de la prueba t Student dado el cumplimiento de la normalidad evaluada con la prueba Kolmogorov-Smirnov con corrección de Lilliefors. Adicional a ello, para las comparaciones con las variables cualitativas se estimaron razones de prevalencias y de odds.

Para identificar las variables de confusión y posibles predictores del inicio de la vida sexual en los adolescentes del estudio se realizó regresión logística binaria multivarible, cuya bondad de ajuste se determinó con el estadístico de Hosmoer-Lemeshow.

Entre los estudiantes que habían iniciado su vida sexual se describieron las razones para utilizar el condón y las prácticas relacionadas con su uso a través de proporciones con sus intervalos de confianza y se determinó su asociación estadística con zona de residencia, el grado escolar, el sexo, el estrato social, el tipo de familia y la fuente de información 
en SSR a través de pruebas Z de comparación de proporciones y chi cuadrado de Pearson.

Los análisis se realizaron en SPSS 21 y Epidat 3.0 con una significación del 0,05.

\section{Aspectos éticos:}

El proyecto cuenta con aval del Comité de Bioética de la Universidad Cooperativa de Colombia. Este estudio se clasifica como una investigación sin riesgo pues no se realiza ninguna intervención o modificación intencionada de las variables biológicas, fisiológicas, sicológicas o sociales de los individuos que participan en el estudio, ni se tratarán aspectos sensitivos de su conducta. Además se firmó el consentimiento informado para los mayores de 17 años y el asentimiento para los menores 18 años.

\section{Resultados}

la mayor proporción de estudiantes procedía de la zona centro oriental, el 39,7\% fueron hombres, $50,7 \%$ de estrato socioeconómico bajo, 52,5\% de undécimo, 60,4\% del grupo etario de adolescencia tardía; sólo un $28,8 \%$ no participa de grupos sociales, las principales fuentes de información en salud sexual y reproductiva fueron internet y el hogar, sólo el 42,6\% viven en familias nucleares. La edad presentó una media de 15,8 años con rango intercuartil entre 15 y 16; la edad promedio de los padres fue 45,3 y de las madres 41,4; los años promedio de estudio de los padres fueron 9 (Tabla 1).

La prevalencia global de adolescentes que han iniciado su vida sexual fue $54,6 \%$ (Tabla 1 ). La edad promedio de inicio de la vida sexual fue 14,3 años con rango intercuartil entre 13 y 15 años; el número promedio de compañeros sexuales fue 1,25 con rango intercuartil entre 0 y 2 ; el 2,0\% tiene hijos (0,2\% dos y $1,8 \%$ un hijo).
La prevalencia de estudiantes que han tenido relaciones sexuales presentó asociación estadística con la zona de residencia, el sexo, el grado escolar, el grupo etario, el tipo de familia y las fuentes de información en SSR; la prevalencia fue estadísticamente menor en todos las zonas en comparación con la Nororiental, en hombres fue $27 \%$ mayor frente a las mujeres, $16 \%$ en los estudiantes de undécimo, $41 \%$ mayor en adolescencia tardía, 7\% mayor en estudiantes monoparentales frente a los individuos con hogares nucleares; en las fuentes de información se halló que en quienes buscan información sobre SSR en internet el riesgo de iniciar vida sexual se incrementa en un $9 \%$ y el recibir información de amigos en un $10 \%$, mientras que los estudiantes que reciben tal información en sus hogares y colegio presentaron una probabilidad $14 \%$ y $10 \%$ menor respectivamente, de iniciar su vida sexual durante la adolescencia (Tabla 2). Adicional a esto se halló asociación con la edad de la madre y el tener vida sexual activa (Valor p t Student=0,049).

En la tabla 3 se corroboró que las asociaciones halladas en el análisis bivariado se conservan en el ajuste multivariado evidenciando que éstas constituyen buenos predictores del riesgo de iniciar vida sexual en adolescentes de décimo y undécimo, con excepción del grado escolar, el tipo de familia y el recibir información en SSR a través de internet.

La prevalencia de estudiantes que han tenido relaciones sexuales no presentó asociación estadística con el estrato socioeconómico $(53,9 \%$ en el bajo, $55,6 \%$ en el medio y $54,7 \%$ en el alto, $\mathrm{Vp}$ $\left.\mathrm{Chi}^{2}=0,639\right)$, ni con la participación en grupos sociales $(54,3 \%$ en quienes pertenecen a grupos sociales y $55,4 \%$ en quienes no lo hacen; $\left.\mathrm{Vp} \mathrm{Chi}^{2}=0,579\right)$, ni con el recibir información sobre SSR por la televisión (56,1\% en quienes si usan este medio y $54,5 \%$ en quienes no lo hacen, $\left.\mathrm{Vp} \mathrm{Chi}^{2}=0,583\right)$. De la misma forma, no se halló asociación con la edad del padre (Valor p t Student=0,243), la escolaridad de la ma- 
Tabla 1. Descripción socio-demográfica y prevalencia de conductas sexuales de riesgo del grupo de estudio

\begin{tabular}{|c|c|c|c|}
\hline & Factores & $\%(\#)$ & IC $95 \%$ \\
\hline \multirow[t]{7}{*}{ Sector de residencia } & NorOriental & $6,5(226)$ & $5,7-7,4$ \\
\hline & NorOccidental & $17,1(590)$ & $15,8-18,4$ \\
\hline & Centro Oriental & $27,5(951)$ & $26,0-29,1$ \\
\hline & Centro Occidental & $8,0(276)$ & $7,1-8,9$ \\
\hline & Sur Oriental & $13,6(469)$ & $12,4-14,7$ \\
\hline & Sur Occidental & $9,9(342)$ & $8,8-10,9$ \\
\hline & Corregimientos & $17,3(599)$ & $16,1-18,6$ \\
\hline \multirow[t]{2}{*}{ Sexo } & Hombre & $39,7(1372)$ & $38,1-41,4$ \\
\hline & Mujer & $60,3(2081)$ & $58,6-61,9$ \\
\hline \multirow{3}{*}{$\begin{array}{l}\text { Estrato socioeconómico de } \\
\text { la vivienda }\end{array}$} & Bajo (1-2) & $50,7(1750)$ & $49,0-52,4$ \\
\hline & Medio (3-4) & $42,2(1458)$ & $40,6-43,9$ \\
\hline & Alto $(>4)$ & $7,1(245)$ & $6,2-8,0$ \\
\hline \multirow[t]{2}{*}{ Grado } & Décimo & $52,5(1812)$ & $50,8-54,2$ \\
\hline & Undécimo & $47,5(1641)$ & $45,8-49,2$ \\
\hline \multirow[t]{2}{*}{ Grupo de adolescencia } & Temprana (10-14) & $39,6(1365)$ & $37,9-41,2$ \\
\hline & Tardía (15-19) & 60,4 (2085) & $58,8-62,1$ \\
\hline \multirow{2}{*}{$\begin{array}{l}\text { Participación en grupos } \\
\text { sociales }\end{array}$} & $\mathrm{Si}$ & $71,2(2460)$ & $69,7-72,8$ \\
\hline & No & 28,8 (993) & $27,2-30,3$ \\
\hline \multirow{5}{*}{$\begin{array}{l}\text { Fuentes de información } \\
\text { en salud sexual }\end{array}$} & Internet & 55,6 (1919) & $53,9-57,2$ \\
\hline & Hogar & $52,4(1808)$ & $50,7-54,0$ \\
\hline & Amigos & $43,3(1497)$ & $41,7-45,0$ \\
\hline & Colegio & $22,9(790)$ & $21,5-24,3$ \\
\hline & Televisión & $9,0(310)$ & $8,0-9,9$ \\
\hline \multirow[t]{4}{*}{ Tipo de familia } & Nuclear & $42,6(1472)$ & $41,0-44,3$ \\
\hline & Monoparental & $40,7(1404)$ & $39,0-42,3$ \\
\hline & Extensa & $10,8(372)$ & $9,7-11,8$ \\
\hline & Otro & $5,9(205)$ & $5,1-6,7$ \\
\hline \multirow{3}{*}{$\begin{array}{l}\text { Ha tenido relaciones } \\
\text { sexuales }\end{array}$} & $\mathrm{Si}$ & $54,6(1887)$ & $53,0-56,3$ \\
\hline & No & $45,3(1566)$ & $43,7-47,0$ \\
\hline & $\begin{array}{c}\text { Media } \pm \\
\text { Desviación estándar }\end{array}$ & $\begin{array}{c}\text { Mediana } \\
\text { (Rango Intercuartil) }\end{array}$ & Mínimo - Máximo \\
\hline Edad & $15,8 \pm 1,0$ & $16(15-16)$ & $13-20$ \\
\hline Edad madre & $41,4 \pm 6,46$ & $41(36-45)$ & $25-78$ \\
\hline Edad padre & $45,3 \pm 7,4$ & $45(40-50)$ & $29-96$ \\
\hline Escolaridad padre & $8,7 \pm 3,2$ & $10(6-11)$ & $0-19$ \\
\hline Escolaridad madre & $9,4 \pm 3,1$ & $11(7-11)$ & $0-20$ \\
\hline
\end{tabular}

IC 95\%: Intervalo de confianza del 95\% para una proporción. 
Tabla 2. Relación de las prácticas sexuales con las condiciones demográficas económicas y sociales.

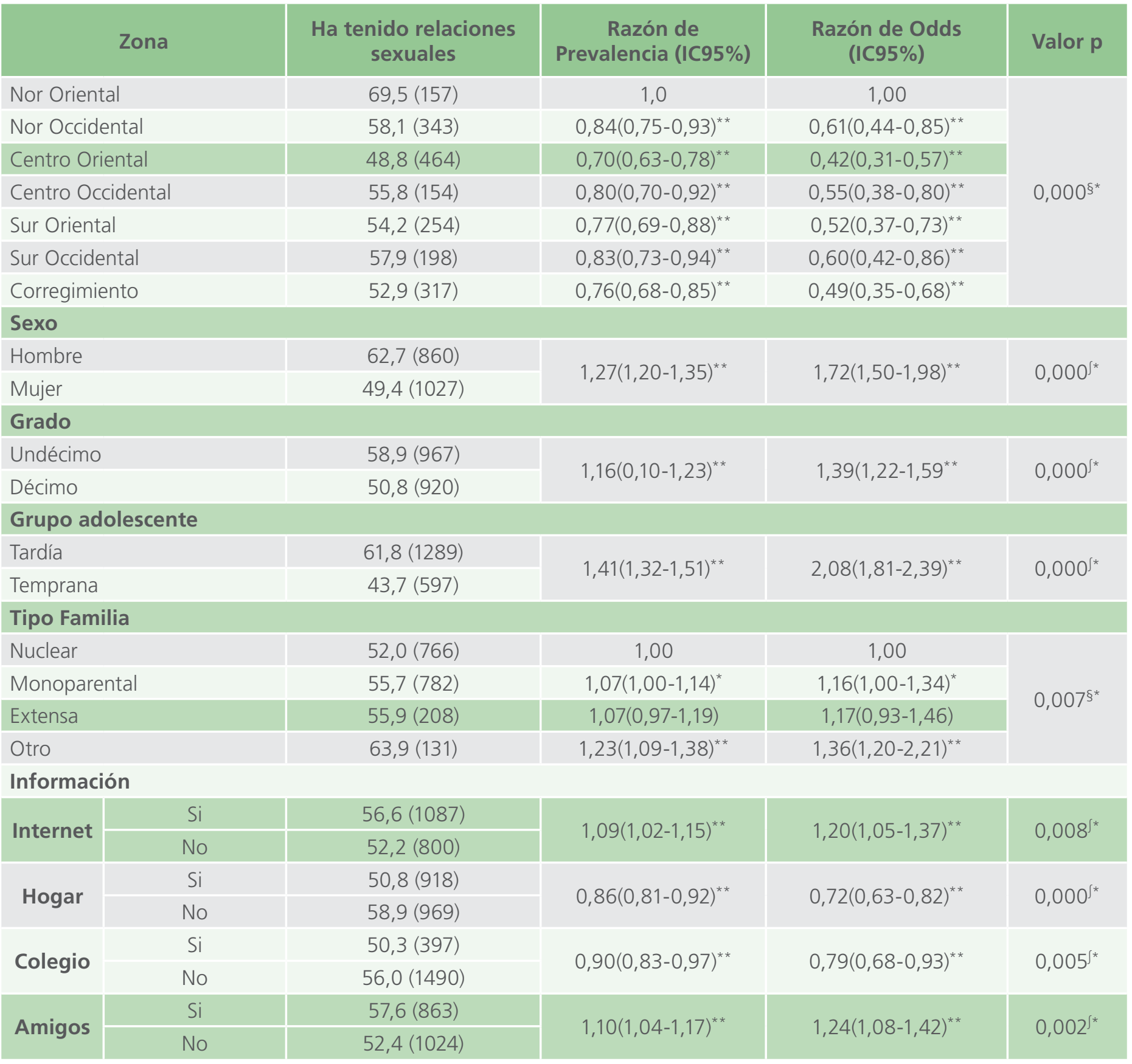

$\S$ Chi cuadrado de Pearson.

S Prueba Z de comparación de proporciones.

* El estadístico es significativo en el 0,01. 
Tabla 3. Modelo de regresión logística multivariable para la prevalencia de relaciones sexuales.

\begin{tabular}{|l|c|c|c|c|}
\multicolumn{1}{c|}{ Variables del modelo } & \multirow{2}{*}{ Zolor $\mathbf{p}$} & \multirow{2}{*}{ Razón de Odds } & \multicolumn{2}{c|}{ IC 05\% Razón de Odds } \\
\cline { 5 - 6 } & & & Inferior & Superior \\
\hline NorOccidental & 0,038 & $0,698^{*}$ & 0,497 & 0,980 \\
\hline Centro Oriental & 0,016 & $0,660^{*}$ & 0,471 & 0,924 \\
\hline Centro Occidental & 0,033 & $0,658^{*}$ & 0,448 & 0,966 \\
\hline SurOriental & 0,002 & $0,570^{* *}$ & 0,401 & 0,809 \\
\hline SurOccidental & 0,077 & $0,716^{*}$ & 0,494 & 1,037 \\
\hline Corregimientos & 0,001 & $0,566^{* *}$ & 0,403 & 0,795 \\
\hline Sexo Hombre/Mujer & 0,000 & $1,686^{* *}$ & 1,428 & 1,990 \\
\hline Grupo de adolescencia & 0,000 & $2,053^{* *}$ & 1,777 & 2,373 \\
\hline Tardía/Temprana & & & & 1,027 \\
\hline Edad de la madre & 0,007 & $1,016^{* *}$ & 1,004 & \\
\hline Fuente de información en sexualidad Si/No & & & & 0,896 \\
\hline Hogar & 0,001 & $0,777^{* *}$ & 0,674 & 0,893 \\
\hline Colegio & 0,001 & $0,753^{* *}$ & 0,635 & 1,508 \\
\hline Amigos & 0,000 & $1,304^{* *}$ & 1,128 & \\
\hline
\end{tabular}

*El estadístico es significativo en el 0,05. **El estadístico es significativo en el 0,01.

La categoría de referencia en la zona de residencia fue la NorOriental.

dre (Valor p t Student=0,725), ni la escolaridad del padre (Valor p t Student=0,884).

En los estudiantes que han iniciado su vida sexual se halló una frecuencia de uso del condón del 66,3\%, aunque sólo el 38,3\% lo usa en todas sus relaciones sexuales y más de la mitad lo utilizan en momentos incorrectos. En este subgrupo las principales razón para no utilizar el condón se relacionan con la creencia que "disminuye el placer sexual", el usar otros métodos de planificación y tener pareja estable (Tabla 4).

Cabe aclarar que las razones citadas para no usar el condón no presentaron diferencias estadísticas según la zona de residencia, el grado escolar, el sexo, el estrato social, el tipo de familia, ni la fuente de información en SSR; con excepción del tener pareja estable y el usar otro método de planificación que fueron razones indicadas con mayor frecuencia por las mujeres (pareja estable en el 26,0\% de las mujeres y $17,4 \%$ de los hombres; uso de otro método $35,5 \%$ de las mujeres y $21,0 \%$ de los hombres).

\section{Discusión}

En esta población la principal fuente de información en sexualidad fue internet, cerca de la mitad de los adolescentes viven en familias nucleares con madre como cabeza de hogar, un poco más de la mitad ha iniciado su vida sexual, siendo los principales grupos de riesgo los hombres, de adolescencia tardía, provenientes de familias monoparentales y en quienes buscan información sobre salud sexual en internet o amigos; esto permite focalizar las acciones de educación en salud sexual y reproductiva en los grupos de mayor riesgo, enfatizando en la importancia del enfoque de género. Además, se debe 
Tabla 4. Actitudes y Prácticas frente al uso del condón en estudiante que han tenido relaciones sexuales.

\begin{tabular}{|c|c|c|c|}
\hline$N=1887$ & Factores & $\%$ & IC $95 \%$ \\
\hline \multirow{15}{*}{$\begin{array}{l}\text { Razones para no } \\
\text { utilizar el condón }\end{array}$} & Disminución del placer & $48,3(911)$ & $46,0-50,6$ \\
\hline & No lo tenía & $44,1(833)$ & $41,9-46,4$ \\
\hline & Uso de otro método & $28,9(546)$ & $26,9-31,0$ \\
\hline & Pareja estable & $22,1(417)$ & $20,2-24,0$ \\
\hline & Confianza en la pareja & $19,8(374)$ & $18,0-21,6$ \\
\hline & Condón no protege & $17,4(329)$ & $15,7-19,2$ \\
\hline & Vergüenza comprarlo & $15,2(287)$ & $13,6-16,9$ \\
\hline & Padres se enojarían & $15,4(291)$ & $13,8-17,1$ \\
\hline & No le gusta & $12,5(235)$ & $11,0-14,0$ \\
\hline & Objeción de la pareja & $7,8(147)$ & $6,5-9,0$ \\
\hline & Se rompe & $7,3(138$ & $6,1-8,5$ \\
\hline & Evitar interrumpir el acto sexual & $6,9(130)$ & $5,7-8,1$ \\
\hline & Da vergüenza usarlo & $5,9(112)$ & $4,8-7,0$ \\
\hline & Mantener el romanticismo & $5,5(103)$ & $4,4-6,5$ \\
\hline & Evitar dudas en la pareja & $5,2(98)$ & $4,2-6,2$ \\
\hline \multirow[t]{9}{*}{ Uso del condón } & \multicolumn{3}{|l|}{ Frecuencia de uso } \\
\hline & Siempre & $38,3(723)$ & $36,1-40,5$ \\
\hline & Frecuentemente & $16,5(311)$ & $14,8-18,2$ \\
\hline & Ocasionalmente & $31,8(600)$ & $29,7-33,9$ \\
\hline & Nunca & $13,4(253)$ & $11,8-15,0$ \\
\hline & \multicolumn{3}{|l|}{ Momento del uso } \\
\hline & Erección & $41,2(778)$ & $39,0-43,5$ \\
\hline & Penetración & $24,4(460)$ & $22,4-26,3$ \\
\hline & Antes de la eyaculación & $34,3(648)$ & $32,2-36,5$ \\
\hline
\end{tabular}

IC 95\%: Intervalo de confianza del 95\% para una proporción.

tener presente que el elevado riesgo descrito para los grupos anteriores se exacerba al tener presente que la frecuencia de uso del condón fue $66,3 \%$ y entre quienes lo utilizan sólo el 38,3\% lo hace en todas sus relaciones sexuales.

En adolescentes escolarizados de Medellín se halló un $54,6 \%$ de adolescentes que presentan riesgo sexual en tanto tienen vida sexual activa, en esta población las fuentes de información sobre salud sexual y reproductiva, la zona de residencia, la edad y el sexo juegan un papel importante como posibles factores explicativos de las conductas sexuales. Resultados similares respecto a las variables que influyen en el comportamiento sexual de riesgo en los adolescentes fueron reportados por el grupo de García en cuya investigación proponen la necesidad de disminuir el grado de riesgo de esta población 
a partir de programas de educación sexual ${ }^{14}$. Vale aclarar que esta investigación también evidenció la relevancia de otras variables de riesgo sexual como la impulsividad, la inmediatez y la vulnerabilidad inherentes a esta etapa de la vida ${ }^{14}$.

La prevalencia de relaciones encontradas en estos adolescentes fue de $54,6 \%$, lo que resulta similar a un estudio sistemático sobre prevalencia de relaciones sexuales en adolescentes colombianos, donde se estimó que el inicio de estas varía entre $7.7 \%$ y $58.0 \%$. Además de ello varios estudios de los que se reportan allí hallaron que la edad media inicio de las relaciones sexuales es de los 14 años. Lo anterior resulta de la percepción de riesgo que tenga el adolescente y de algunas condiciones propias como la edad, sexo, educación, grupo cultural y/o religioso, entre otras. ${ }^{15}$

En la población estudiada se identificó un mayor riesgo en los hombres, lo que podría suponer que cultural y socialmente en este subgrupo se ha fomentado el inicio precoz de la vida sexual como medio para demostrar su virilidad, aunque también podría suponer presión por los pares, impulso biológico y búsqueda de sensaciones sexuales ${ }^{16}$. En contraste, algunos autores han indicado que las mujeres comprenden las implicaciones que tiene el inicio de la vida sexual como lo es un embarazo no deseado, además de ello algunas condiciones socio-culturales pueden vetar conductas relacionadas con la sexualidad en este grupo ${ }^{17}$. No obstante, otros han referido una nueva tendencia en la actividad sexual por género, puesto que años atrás al género masculino se le atribuía inicio precoz de relaciones, más parejas sexuales y ocasionales, y corta duración de relaciones sentimentales; lo que en algunos contextos se ha extendido al grupo de mujeres ${ }^{18}$.

En relación con la edad se observó un mayor número de relaciones sexuales en el grupo de individuos pertenecientes a la adolescencia tardía, lo que coincide con el mayor riesgo detectado para los de grado undécimo; esto es similar a lo reportado en el estudio de Castro en el cual se concluyó que el inicio de vida sexual y el tener vida sexual activa es mayor en los grados escolares más altos ${ }^{16}$. Sin embargo, esto difiere con lo encontrado por Ceballos donde se explica que las relaciones se presentan cada vez a menor edad, y se puede atribuir a la mayor disponibilidad de información sobre sexualidad en diferentes medios ${ }^{18}$.

Con respecto a los medios de información sobre salud sexual y reproductiva se encontró que el recibir información de internet contribuye a un mayor riesgo sexual, en contraste con recibirla en el colegio o el hogar. Esto resulta coherente con textos previos que refieren cómo en la era de la informática los adolescentes tienen fácil acceso a información en internet con un subsecuente uso inapropiado que ha derivado en conductas adictivas y de riesgo por el tipo de contenidos a los cuales se puede acceder ${ }^{19}$. Respecto a la familia se ha evidenciado el rol protagónico que tiene al brindar información y formación relacionada con sexualidad, aunque la calidad de los contenidos en este tema podrían estar afectados el tipo de comunicación y el grado de confianza en las relaciones con los adolescentes, en este sentido vale precisar que en algunos contextos los jóvenes refieren su interés en que los padres sean la primera fuente de información en salud sexual y reproductiva, mientras que en otros escenarios se ha indicad que los padres presentan temores frente a esta temática por creer que los conocimientos sobre sexualidad hacen proclives a los adolescentes a prácticas de riesgo sexual10,20-22.

Entre los adolescentes que ya han iniciado su vida sexual se registró frecuencia de uso del condón de $66,3 \% ; 58,7 \%$ lo utilizan en el momento correcto y sólo 38,3\% lo usa en todas sus relaciones sexuales. A pesar de que se ha documentado la eficacia del condón como método de prevención de enfermedades de transmisión sexual y del embarazo, los jó- 
venes poseen un bajo conocimiento sobre la forma correcta del uso del condón, dentro de los cuales podemos destacar que desconocen que deben usar el condón desde el momento de la erección y durante toda la relación sexual ${ }^{23}$.

La disminución del placer sexual, la no disponibilidad del preservativo al momento del coito y estar utilizando otro método de planificación, son las principales razones por las cuales los adolescentes en algún momento han dejado de lado el uso del condón. Resultados similares fueron reportados por García R donde adicional a lo anterior existían dificultades por incomodidad, temor al rechazo de la pareja y objeción de la misma24. Estos comportamientos de elevado riesgo hacen a los adolescentes una población vulnerable a las infecciones de transmisión sexual y otros problemas relacionados con su salud sexual y reproductiva ${ }^{25}$.

Se debe tener presente que el uso apropiado del condón constituye una de las principales medidas de protección frente a las ITS y los embarazos no deseados, y en esta población de Medellín su forma y frecuencia de uso resultó muy baja, lo que exacerba el riesgo sexual de los adolescentes. En este sentido se debe indicar que pocas investigaciones se han focalizado en los mitos y prácticas relacionadas con el uso del preservativo, particularmente en la evaluación de su uso correcto, lo que brinda opciones para investigaciones posteriores que busquen profundizar en este tema 26 .

Entre las limitaciones del estudio está el sesgo temporal y el carácter exploratorio de las asociaciones estadísticas; no obstante, esta investigación presenta varias ventajas como el ser uno de los pocos estudios representativo de un elevado número de adolescentes de la ciudad, lo que le brinda gran validez externa para orientar diferentes programas y acciones de salud y política pública para la salud sexual del adolescente.

\section{Conclusión}

El elevado riesgo sexual, la elevada frecuencia de mitos sobre el condón y la identificación de grupos de mayor vulnerabilidad sexual, constituyen resultados de gran utilidad para formular, implementar o evaluar las políticas públicas relacionadas con la adolescencia y la salud sexual y reproductiva.

\section{Financiación}

Universidad de Antioquia. Universidad Cooperativa de Colombia, Convocatoria General 2013.

\section{Conflicto de intereses}

Ninguno de los autores declara conflicto de interés para la publicación de este manuscrito.

\section{Referencias}

1. Profamilia. Adolescencia. [Internet] 2013. [consultado 10 Ago 2014]. Disponible en: http://profamilia.com/index. php?option=com_content $\&$ view $=$ article $\&$ id $=337 \% 3$ Acosasque-pasan-en-la-adolescencia\&catid=55\&ltemid $=250$.

2. Páramo M. Factores de riesgo y factores de protección en la adolescencia: Análisis de contenido a través de grupos de discusión. Terapia Psicológica. 2011;29(1):85-95.

3. Organización Mundial de la Salud, Fondo de Población de las Naciones Unidas, UNICEF. Actividades a favor de la salud de los adolescentes. Hacia un programa común. [Internet] 1997. [consultado 10 Ago 2014]. Disponible en: http://whqlibdoc. who.int/hq/1997/WHO_FRH_ADH_97.9_spa.pdf?ua=1.

4. Soto $V$. Factores asociados al no uso del condón. Estudio en adolescentes y adultos jóvenes de Chiclayo. Anales de la Facultad de Medicina. 2006;67(2):152-9.

5. Cossio P. Factores psicosociales asociados a conductas de riesgo de una población de adolescentes de bachilleratos. Episteme [Internet] 2005. [consultado 10 Ago 2014]. Disponible en: http://www.uvmnet.edu/investigacion/episteme/numero2-05/ impresiones/a_factores.asp.

6. Failde J, Lameiras M, Bimbela J. Prácticas sexuales de chicos y chicas españoles de 14-24 años de edad. Gaceta Sanitaria. 2008;22(6):511-9. 
7. Rodríguez L, Suárez N. Conocimiento, actitud y el uso del condón masculino en población de riesgo de contraer VIH/SIDA Cuba 2005. Horizonte Sanitario. 2006;5(1):12-27.

8. Noguerol M. Uso del preservativo en adolescentes como método de prevención de enfermedades. [Internet] Pediatrics. 2013;132;973. [consultado 10 Ago 2014]. Disponible en: http:// www.intramed.net/contenidover. asp?contenidoID $=82841$.

9. González J. Conocimientos, Actitudes y Prácticas sobre la sexualidad en una población adolescente escolar. Revista de Salud Pública. 2009;11(1):14-26.

10. Cardona J, Carmona P, Rosero C. Conocimientos, actitudes y prácticas acerca de infecciones de transmisión sexual en adolescentes del municipio de Caldas (Colombia). Salud Uninorte. 2013;29(2):236-48.

11. Romero M, Piedrahita C, Ochoa M, Vargas C. Condón: uso y creencias en la población del centro de Medellín en 2005. Revista Salud Pública de Medellín. 2008;3(1):37-46.

12. Alvis N, Mattar S, García J, Conde E, Díaz A. Infecciones de Transmisión Sexual en un grupo de alto riesgo de la ciudad de Montería, Colombia. Rev. Salud Pública. 2007;9(1):86-96.

13. Gómez R, Herrera A, Herrera A, Delgado A, Mejía C, Vanegas $A$, et al. Conocimientos mitos y creencias frente al condón y la sexualidad en jóvenes escolarizados de Pereira. Revista Médica de Risaralda. 2001;7(1):9-14.

14. García E, Menéndez E, Fernández P, Cuesta M. Sexualidad, anticoncepción y conducta sexual de riesgo en adolescentes. International Journal of Psychological Research. 2012;5(1):7987.

15. Campo A. Relaciones sexuales en adolescentes colombianos y las implicaciones para la salud pública: una revisión de la prevalencia y algunas variables asociadas Medunab 2009; 12(2), 86.

16. Castro I. Conocimientos y factores de riesgo sobre infecciones de transmisión sexual en adolescentes. Revista Habanera de Ciencias Médicas. 2010;9(3):705-16.

17. Campo A, Silva J, Meneses M, Castillo M, Navarrete P. Factores asociados con el inicio temprano de relaciones sexuales en estudiantes adolescentes de un colegio de Bucaramanga, Colombia. Revista Colombiana de Psiquiatría. 2004;33(4):36777.

18. Ceballos G, Campo A. Prevalencia de relaciones sexuales en estudiantes de secundaria entre 10 y 12 años Santa Marta Colombia. Revista Facultad de Medicina de la Universidad Nacional de Colombia. 2006;54(1):4-11.

19. Rial A, Gómez P, Braña T, Varela J. Actitudes, percepciones y uso de internet y las redes sociales entre los adolescentes de la comunidad gallega (España). Anales de Psicología. 2014;30(2):642-55.

20. Pérez Z, Casas L, Peña L, Miranda O, Zaldívar M. Información sexual en un grupo de adolescentes. [Internet] Revista Cubana de Medicina Militar. 2002;31(4). [Consultado 14 de Ago de 2014]. Disponible en: http://www.bvs.sld.cu/revistas/mil/vol31_4_02/ mil060402.htm.
21. Romero R, Lora $M$, Cañete R. Adolescentes y fuentes de información de sexualidad: preferencias y utilidad percibida. Atención Primaria. 2001;27(1):12-17.

22. Torriente N, Diago D, Rizo A, Menéndez L. Conocimientos elementales sobre educación sexual en alumnos de una escuela secundaria básica urbana. Revista Habanera de Ciencias Médicas. 2010;9(4):576-87.

23. Badillo, M. C. C., Figuera, F. A. C., \& Castro, B. Z. (2012). Prácticas inadecuadas del uso del condón y factores asociados en estudiantes universitarios. Revista Salud UIS. 2012; 43(3).

24. García R, Cortés A, Vila L, Hernández M, Mesquia A. Comportamiento sexual y uso del preservativo en adolescentes y jóvenes de un área de salud. [Internet] Revista Cubana de Medicina General Integral. 2006;22(1). [Consultado 14 de Ago de 2014]. Disponible en: http://bvs.sld.cu/revistas/mgi/ vol22_1_06/mgi03106.htm.

25. Lavielle $P$, Jiménez $F$, Vázquez $A$, Aguirre $M$, Castillo $M$, Vega $S$. Impacto de la familia en las conductas sexuales de riesgo de los adolescentes. Revista Médica del Instituto Mexicano del Seguro Social. 2014;52(1):38-43

26. Caricote E. Los docentes y la educación sexual en la adolescencia. Revista de Ciencias de la Educación. 2008;18(32).

\section{Opina sobre este artículo:}

\section{(f) (B) in $8+\boldsymbol{S}$ P}

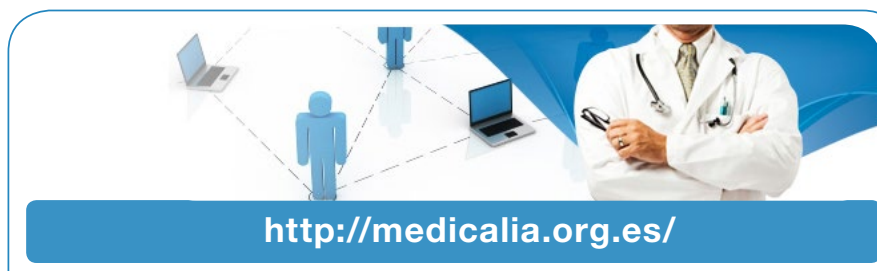

Los médicos disponen de una red social para intercambiar experiencias clínicas, comentar casos y compartir conocimiento. También proporciona acceso gratuíto a numerosas publicaciones. ¡Únase ahora!

\section{Publique con iMedPub}

http://www.imed.pub

\section{Biomedicina}

$\checkmark$ Biomedicina es la primera revista de acceso abierto en Español, dedicada a todas las áreas de la medicina.

$\checkmark$ Recoge artículos de investigación sobre todas las áreas de la medicina. 